\title{
ARTIGO
}

\section{M etodologia de Sistemas Flexíveis aplicada a estudos em Ciência da Informação: uma experiência pedagógica}

\author{
The Soft Systems M ethodology applied \\ to Information Science studies: \\ A pedagogical experience
}

Sely M.S. COSTA $^{1}$

R E S U M O

Este trabalho descreve a aplicação da Metodologia de Sistemas Flexíveis - Soft Systems Methodology a um programa de mudança organizacional no Departamento de Ciência da Informação da Universidade de Brasília. A metodologia em questão foi desenvolvida por Peter Checkland, como alternativa às metodologias de sistemas rígidos, como a pesquisa operacional. Tem sido aplicada nas mais diversificadas situações, entre outras, para projetos relacionados com situações-problema que ocorrem em organizações. Mais recentemente, aplicou-se com freqüência em pesquisas acadêmicas na área de Ciência da Informação, especialmente na Inglaterra, e agora, no Brasil, onde o Soft Systems Methodology foi introduzido como disciplina específica do programa de pós-graduação do Departamento de Ciência da Informação da Universidade de Brasília e da orientação de teses e dissertações. Este trabalho discute a Soft Systems Methodology e seus fundamentos, assim como a experiência de seu ensino na pós-graduação e sua aplicação em problemas da Ciência da Informação. A Soft Systems Methodology consiste de sete estágios

\footnotetext{
1 PhD em Ciência da Informação pela Loughborough University, Inglaterra. Professora Adjunta da UnB. Editora-chefe da Revista de Biblioteconomia de Braślia. Departamento de Ciência da Informação, Universidade de Brasília. Campus Universitário Darcy Ribeiro, Asa Norte, 70910-900 Brasília, DF, Brasil. E-mail: selmar@unb.br

Recebido e aceito para publicação em 8/7/2003.
} 
e faz uso de ferramentas específicas, sendo fundamentada na abordagem sistêmica, na fenomenologia e na Pesquisa-Ação. Este trabalho descreve ainda, a partir do levantamento bibliográfico sobre o Soft Systems Methodology, a organização do conteúdo programático da disciplina, atendendo a alunos de mestrado e doutorado em Ciência da Informação, e a observação sistemática, tanto das percepções dos alunos e suas reações relativas à disciplina durante todo o período letivo, quanto da aplicação da Soft Systems Methodology em situações-problema reais. Incluem-se na discussão as ações desenvolvidas visando melhor interação entre alunos de graduação, bolsistas do PIBIC, e alunos de pós-graduação do citado departamento, cujo perfil educacional e profissional era variado: Engenharia, Ciência da Computação, Ciência da Informação e Administração. A interação entre graduandos e pós-graduandos levou a uma aproximação efetiva entre os dois âmbitos do depto. A metodologia e sua aplicação no programa acadêmico de Ciência da Informação despertaram grande interesse e motivação, além de contribuir para os conhecimentos da área e para sua interdisciplinaridade - não só pelo corpo teórico em que se baseia, como também pela mencionada diversidade dos alunos. Tal diversidade permitiu criar-se uma variedade de situações em que se aplicou a metodologia. Conclui-se que a Soft Systems Methodology é alternativa viável e adequada ao tratamento de diversos problemas, além de contribuir significativamente ao corpo de conhecimentos da Ciência da Informação.

Palavras-chave: Metodologia de sistemas flexíveis, problemas organizacionais, Ciência da Informação, estudos de pós-graduação, interação graduação e pós-graduação.

This paper discusses the Soft Systems Methodology and its fundamentals, as well as the application of the Soft Systems Methodology in a graduate program on organizational change in the Information Science Department at the University of Brasília. The Soft Systems Methodology was developed by Peter Checkland as an alternative to hard systems methodologies, such as operational research. The methodology consists of seven stages and uses specific tools; it's based on a systemic approach, on phenomenology, and hands-on research. It has been applied to the most diverse situations, including problem-situations in organizations. Soft Systems Methodology has also been applied in Information Science' academic research, particularly in England, and now, in Brazil, at University of Brasilia, where it was established as a discipline in the Information Science Department's graduate program. To establish such discipline, the first step taken was the bibliographic survey on Soft Systems Methodology, followed by the syllabus development and course organization (taking into account the graduate students, but also including the undergraduate, the ones on PIBIC scholarships). The experience included careful observation of the students perceptions and reactions to the subject during the given academic term, as well 
as observation of the Soft Systems Methodology application to real problem-situations. Besides, effective action was taken to improve graduate and undergraduate students' interaction - resulting in a more effective interaction in the dept. as a whole. Applied to the Information Science academic studies, Soft Systems Methodology contributed to that field of knowledge and to its interdisciplinary connections as well, be it for the methodology's theoretical content itself, be it for the students' variety of educational and professional profiles (Engineering, Computer and Information Sciences, Administration). Such students' diversity allowed for a variety of real situations, on which to apply the Soft Systems Methodology. The methodology raised interest and motivation among students, and proved to be a viable and effective alternative, contributing consistently to the improvement of Information Science knowledge.

Key words: Soft Systems Methodology, Information Science, graduate studies, organizational problems, undergraduate and graduate interaction, Universidade

\section{N T R O D U Ç Ã O}

A Metodologia de Sistemas Flexíveis (Soft Systems Methodology-SSM) foi desenvolvida por uma equipe de pesquisadores da Universidade de Lancaster, na Inglaterra. A principal motivação para seu desenvolvimento nasceu da constatação, por parte daqueles pesquisadores, de que as Metodologias de sistemas rígidos (hard) não respondiam de maneira satisfatória à análise de problemas que, tipicamente, ocorrem em organizações dos mais variados tipos. Isto é: a idéia que permeou o desenvolvimento e a aplicação da Metodologia foi a de que os conhecimentos de áreas como a Engenharia de Sistemas não eram apropriados para o estudo de tais problemas.

Não parece haver dúvidas de que as observações daqueles pesquisadores são reflexo das mudanças paradigmáticas que já vinham sendo observadas em outros contextos, especialmente nas Ciências Sociais. Tais mudanças estão relacionadas com a introdução das idéias resultantes do pensamento reflexivo de Husserl (1970), sobre o que chamou de Fenomenologia ${ }^{2}$, no seio de diferentes escolas de pensamento em diversas disciplinas daquela divisão do conhecimento, nomeadamente a Sociologia, a Antropologia e a Educação, entre outras.

O desenvolvimento da SSM, portanto, resultou da introdução dos princípios da Fenomenologia na Engenharia e Análise de Sistemas praticadas até então por aqueles pesquisadores. Inúmeras iniciativas foram levadas a cabo a partir dessa mudança de paradigma diante dos objetos pesquisados, o que resultou no aprimoramento das idéias inicialmente discutidas e da própria Metodologia.

Na medida em que o trabalho foi realizado em conjunto com estudantes de pós-graduação daquele departamento, o uso da mesma como Metodologia de pesquisa - via de regra pesquisa aplicada - e sua posterior migração para outras

\footnotetext{
2 Escola de pensamento filosófico que, em oposição ao positivismo de Comte, considera que a realidade repousa em nossos pensamentos sobre o mundo, mais do que no mundo propriamente dito. Com isso, Husserl considerava que o filósofo, na sua interpretação da realidade, deveria colocar o mundo real "entre parêntesis", não significando com isso que deva negar que exista um mundo "lá fora", mas que o processo filosófico do pensar sobre a realidade deve ser, na verdade, um estudo de significados. Isto é, um estudo no qual o estudioso vê, clarifica, determina e distingue significados (grifo da autora) (HUSSERL, 1970; CHECKLAND, 1999, p.274).
} 
disciplinas, parece ter sido apenas uma questão de tempo. Assim, ao longo de cerca de quatro décadas de desenvolvimento e aplicação, a SSM vem sendo usada em pesquisas nas diversas áreas do conhecimento, em especial na Inglaterra.

No Brasil a SSM tem sido pouco usada e parece pouco disseminada. Há algumas iniciativas registradas na literatura, mas a observação das mesmas aponta para uma utilização acanhada da Metodologia, considerando-se a complexidade que envolve, quando usada em todo o seu potencial.

O contato com a Metodologia durante estudos de $P h D$ na Inglaterra levou a autora deste trabalho ao estudo mais aprofundado da mesma. Tendo sido utilizada em estágio bastante primitivo daquele trabalho de pesquisa, logo foi identificada como não sendo a Metodologia mais apropriada para o tipo de estudo proposto. No entanto, a aplicação de seus dois primeiros estágios em um exercício reflexivo sobre o contexto da pesquisa então em andamento permitiu a construção do modelo conceitual que fundamentou a discussão teórica de todo o estudo. A partir dessa experiência e da motivação que o conhecimento sobre a Metodologia despertou, decidiu-se pelo aprofundamento dos conhecimentos sobre a mesma e sua posterior disseminação no Brasil, na área de Ciência da Informação, via seu ensino em disciplinas de pós-graduação na área e, por conseguinte, a orientação de teses e dissertações.

As experiências adquiridas pela interação com alunos dessas disciplinas têm permitido sua disseminação não só na Ciência da Informação, mas também nas áreas em que laboram aqueles estudantes, a saber: as Engenharias, a Ciência da Computação, a Administração, a Arquitetura e a Comunicação, entre outras, o que, por seu turno, tem permitido sua aplicação em diferentes contextos. É interessante observar, no entanto, que tais aplicações têm-se dado como exercícios acadêmicos, apenas. O uso da Metodologia em pesquisa acadêmica propriamente dita, isto é, envolvendo situações reais, não permite, até o momento, a divulgação de resultados conclusivos, dado que tais pesquisas estão em fase de desenvolvimento.

O trabalho aqui descrito visa, portanto, a: Dar uma visão geral da Metodologia, com base nos trabalhos publicados por Checkland (1999), Checkland e Scholes (1999), Couprie et al. (2000); e Wilson (2000); Apresentar resultados de aplicações encontrados na literatura internacional e nacional, tanto no contexto de sua aplicação prática em situações encontradas nas organizações, quanto no contexto de sua aplicação como Metodologia de pesquisa em estudos de pós-graduação; Descrever resultados de observações sobre experiências recentes com o ensino da Metodologia no contexto de uma disciplina específica em um programa de pós-graduação em Ciência da Informação no Brasil; Relatar resultados primigênios de sua aplicação em projeto de pesquisa realizado no âmbito de um departamento em uma universidade pública federal, visando a um programa de mudança organizacional com enfoque em questões relacionado com a comunicação científica, a comunicação organizacional, a infra-estrutura tecnológica e a adequação curricular.

Parte-se do pressuposto de que sua disseminação por meio de apresentação de trabalhos em eventos científicos, da publicação de artigos em periódicos especializados etc., deve despertar o interesse de um número maior de estudiosos em diferentes áreas e, consequentemente, seu uso em diversificados contextos. Isso, por sua vez, constitui o objetivo último do trabalho aqui apresentado. 


\section{OPCCÕ S GERAIS SOBRE A SSM}

A SSM se baseia no pensamento sistêmico, constituindo, assim, uma linguagem sistêmica. Neste sentido, é apropriada para estudos em que o problema investigado pode ser definido e analisado como sistema. Mais que isso, é necessário que tal problema possa ser descrito como um tipo específico de sistema: um sistema de atividade humana, definido, de acordo com Checkland (1999) como um sistema nocional, no sentido em que não constitui a descrição de atividades reais, mas construtos intelectuais. Constitui, ainda, um sistema intencional na medida em que expressa alguma atividade humana propositada, a qual pode ser, em princípio, encontrada no mundo real. Assim, sistemas de atividade humana são ideais para uso em debates sobre possíveis mudanças que devem ser introduzidas a uma situação problema do mundo real. Formam um conjunto interativo de subsistemas ou atividades e descrevem, de maneira geral, seres humanos realizando atividades propositadas tais como atividades industriais, sistemas políticos, etc. (Figura 1).

O desenvolvimento da SSM permitiu a criação de uma linguagem específica a dos sistemas de atividade humana. Por meio dessa linguagem determinados tipos de problemas, especialmente aqueles encontrados em organizações, são apropriadamente identificados, descritos e analisados, permitindo a proposição de mudanças que contribuirão para o melhoramento das situações consideradas problemáticas.

A "forma básica" da SSM pode, então, ser ilustrada como na Figura 2, onde se pode observar, de modo bastante genérico, como se dá o processo de análise envolvido na sua aplicação.

O processo de análise envolvido na aplicação da SSM, e ilustrado na Figura 2, pode ser melhor visualizado como na Figura 3, onde o mesmo é apresentado de forma mais detalhada, a fim de que as análises em questão sejam representadas mais claramente.

Observe-se que há dois tipos de análise: uma cultural, em que se busca a identificação de valores, normas, papéis, mecanismos de poder, interações, relacionamentos, etc. A outra lógica, em que se aplicam as sete etapas da Metodologia e que permite, a partir de um exercício inicial de estruturação das questões identificadas na situação em estudo, definir seus subsistemas relevantes. Com base nestes, são definidos modelos conceituais por meio dos quais as ações implícitas nos processos de transformação realizados por esses subsistemas estarão logicamente relacionadas, assim como seus mecanismos de controle. Posteriormente, por meio de comparação dos modelos definidos com a situação problema estruturada, é proposto um conjunto de mudanças possíveis e desejáveis que vão melhorar a situação objeto da análise. É interessante notar que nem sempre todas as etapas são aplicadas em um estudo. Mais que isso, sua aplicação via de regra se dá de forma iterativa, no sentido em que há sempre o retorno a uma ou mais etapas anteriores, tantas vezes quantas seja necessário. A Figura 4 contém o mapa da SSM (análise lógica), tal como proposta por Checkland (1999):

\section{A SSM APLICADA A SITU AÇÕ ES PRÁTICAS E EM PESQUISA ACA DÊM ICA}

Há um variado número de exemplos de aplicação da SSM na literatura, em ambos os tipos de projetos. São incluídos neste trabalho apenas uns poucos que podem ajudar no entendimento das questões relacionadas com o uso da SSM em diferentes contextos.

\footnotetext{
3 Uma visão mais detal hada da metodologia e de seus conceitos, terminologia, ferramentas etc é apresentada em artigo desta autora, publicado na Revista de Biblioteconomia de Brasília, v.26, n.1, jan./jun. 2002 (no prelo).
} 

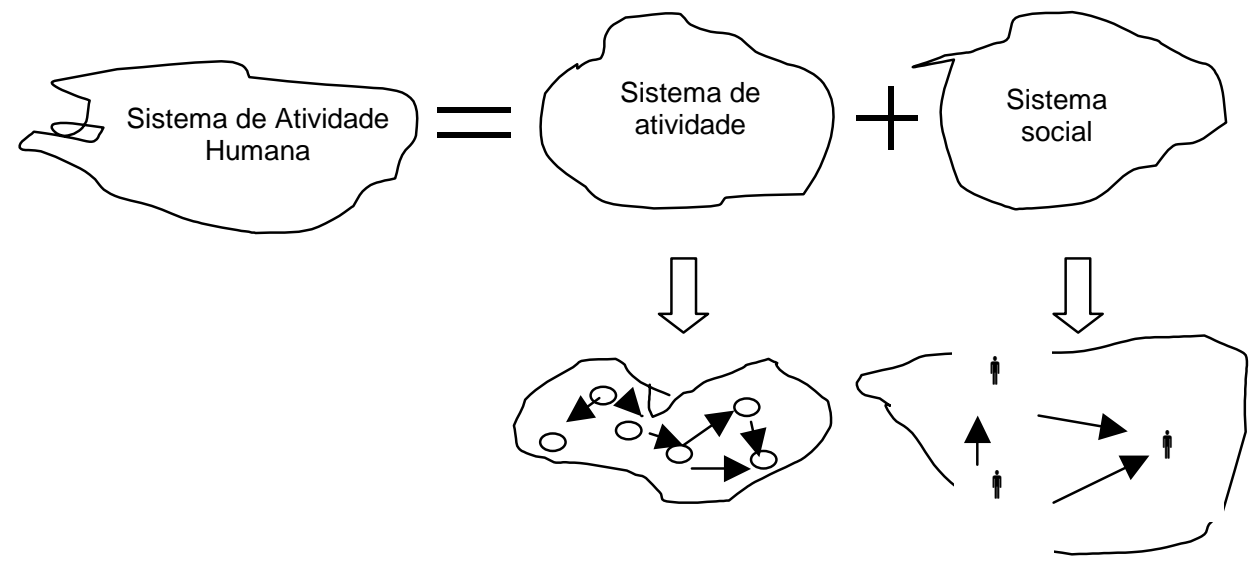

Figura 1. Sistema de Atividade Humana, adaptado de Wilson (2000, p.28).

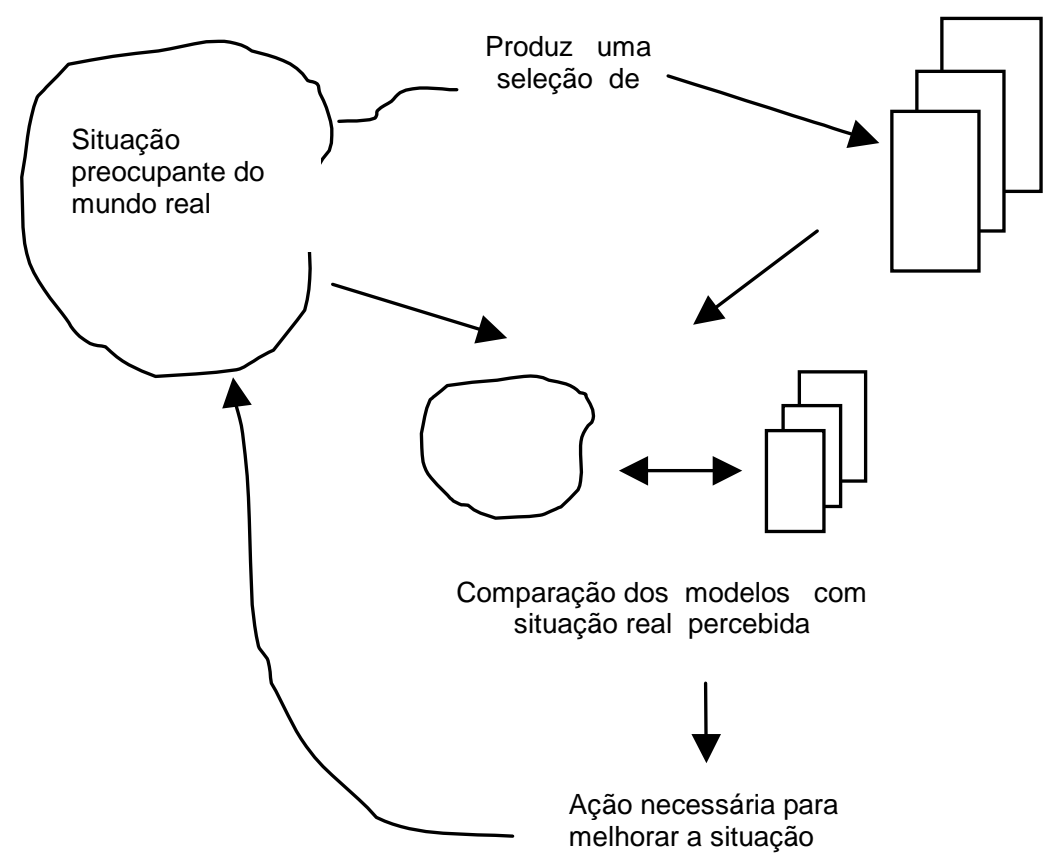

Sistemas relevantes de atividades propositadas

Figura 2. Forma básica da SSM, segundo Checkland e Scholes (1999). 


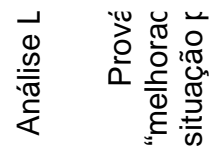
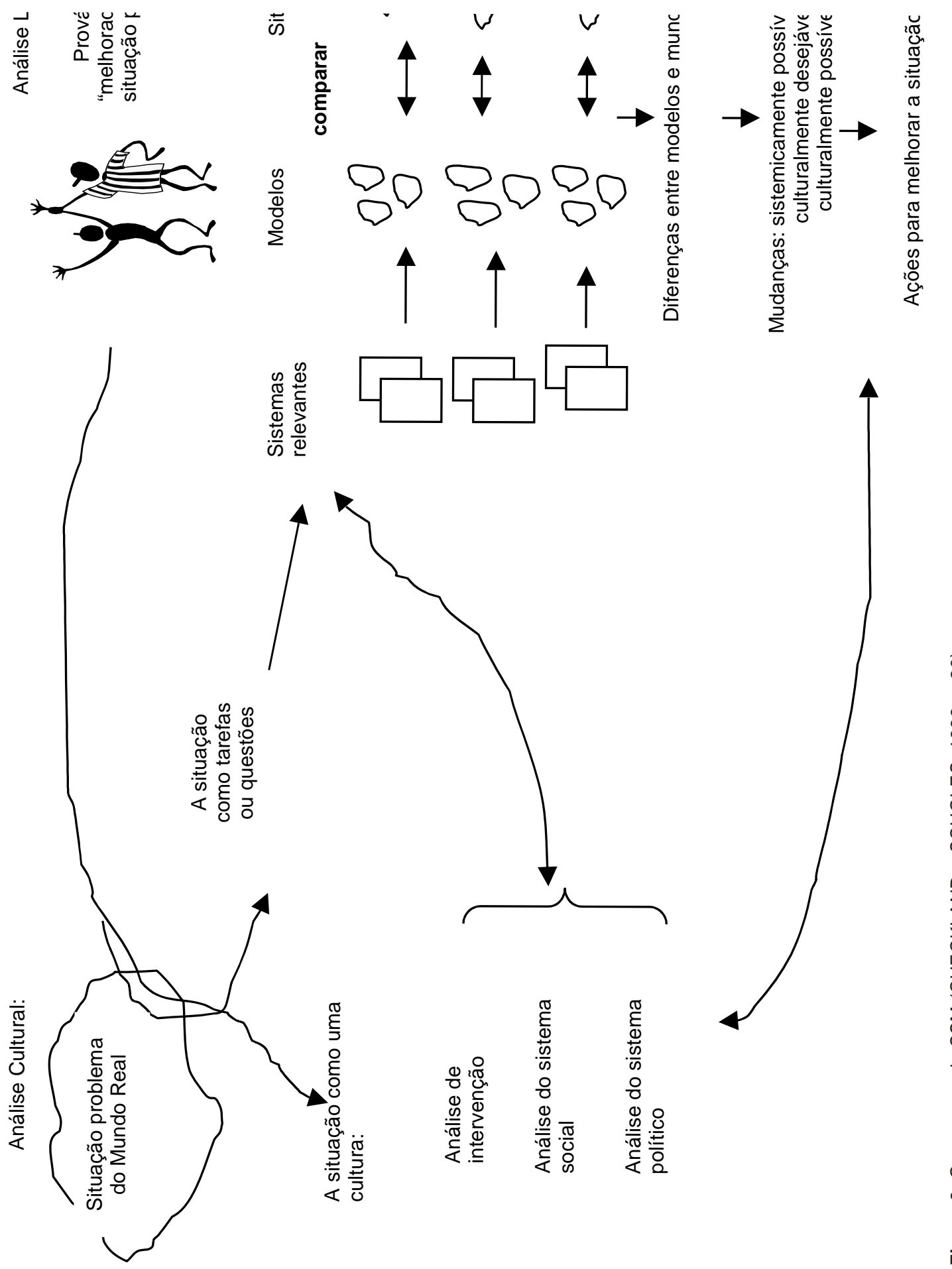

Transinformação, Campinas, 15(2):259-271, maio/ago., 2003 


\section{Aplicações de ordem prática, no exterior}

Checkland e Scholes (1999) divulgaram, em livro, algumas das aplicações realizadas por pesquisadores e estudantes de pós-graduação de Lancaster junto a organizações tanto do setor público quanto do setor privado, no Reino Unido. Embora tais estudos tenham sido realizados no âmbito de um departamento em uma universidade, envolvendo estudantes de pós-graduação e, portanto, constituindo pesquisa acadêmica, os mesmos são incluídos aqui como aplicações práticas em virtude de terem sido realizados "sob encomenda" de instituições públicas e privadas que buscavam algum tipo de melhoria em situações identificadas como problemáticas.

De fato, o relato dos autores visa a, entre outras questões, ilustrar o desenvolvimento e aperfeiçoamento da Metodologia por meio da divulgação desses estudos. Como paradigma da própria Pesquisa-Ação, o que se buscou sempre nos trabalhos desenvolvidos por aqueles pesquisadores e estudantes de pós-graduação se traduz como um processo de aprendizado constante para o qual contribuíram tanto as situações em estudo quanto a própria aplicação
1 A situação problema não estruturada

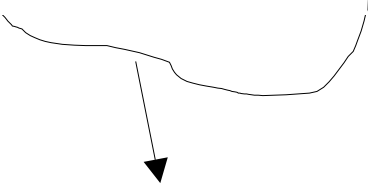

2 A situação problema expressa
7 Ação para melhorar a situação
Mudanças possíveis e desejáveis

5 Comparação de 4 com 2

Mundo rea

Pensamento sistêmico sobre o mundo real

3 Definições fundamentais de subsistemas relevantes

4 Modelos conceituais

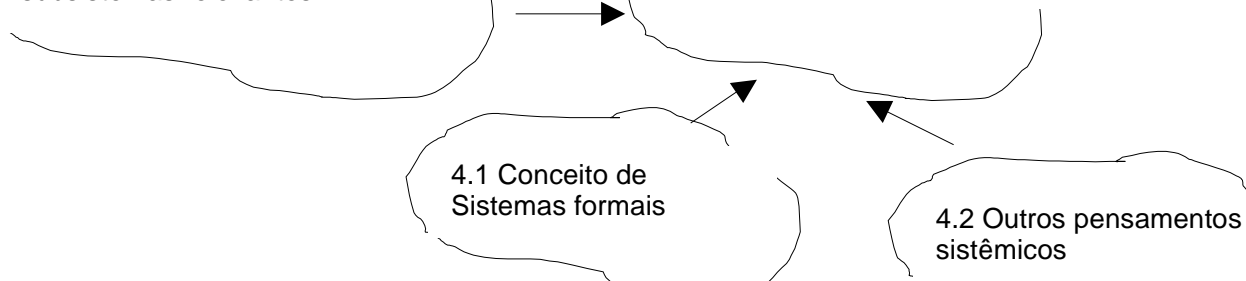

Figura 4. Mapa da Metodologia de Sistemas Flexíveis (CHECKLAND, 1999, p.163). 
da Metodologia. Tais estudos foram realizados em áreas como Saúde Pública, Telecomunicações, Computação, Petróleo, etc. Os resultados obtidos contribuíram para que a Metodologia evoluísse de simples "versão redefinida da engenharia de sistemas" para um trabalho constante de reflexão sobre seus diferentes componentes e ferramentas - sua estrutura -, envolvendo: os três tipos de análise (de intervenção, do sistema social e do sistema político), o CATWOE ${ }^{4}$, a Rich Picture ${ }^{5}$, os três E's (eficiência, eficácia e efetividade), etc. Tais reflexões têm permitido um melhor entendimento tanto da Metodologia como um todo quanto de suas partes constituintes e do ambiente em que se insere, o que, por sua vez, provoca o desenvolvimento do "ciclo hermenêutico de Dilthey", permitindo, por conseguinte, refinados ciclos de ação (CHECKLAND, 1999, p. A5).

Nesta mesma linha de ação, Couprie et al. (2000) da Universidade de Calgary, no Canadá, reportam uma aplicação no departamento de manufaturas de uma empresa do Grupo Shell, que visou a melhoria da capacitação dos empregados alocados naquele departamento, ponto considerado crucial para o melhor desempenho do mesmo. De acordo com o estudo descrito no trabalho de Couprie e seus colegas - o qual foi conduzido pelo próprio Checkland - o uso da SSM permitiu estudar e implementar melhorias no exercício das funções de manufatura de uma das subsidiárias do grupo.

Os trabalhos descritos acima visam dar uma visão bastante geral e sucinta do uso da SSM em situações práticas encontradas no mundo real, nas quais havia o comprometimento com a implementação de fato das melhorias propostas. Outras aplicações, tanto pelo grupo de Lancaster quanto por outras equipes de pesquisadores, vêm, desde então (década de 70 ), sendo realizadas e reportadas na literatura.

\section{Aplicações em pesquisa acadêmica na Ciência da Informação, no exterior}

No campo das pesquisas consideradas aqui como puramente acadêmicas, isto é, não necessariamente comprometidas com a implementação das ações propostas como melhoria das situações estudadas, alguns exemplos de estudos na área de Ciência da Informação ilustram o uso da SSM como Metodologia de pesquisa. Esses exemplos compreendem, aqui, pesquisas de doutorado e mostram a aplicação da Metodologia em três tipos de situação. Primeiro, questões relacionadas com problemas ocorrendo em universidades da Coréia do Sul, relativos a redes eletrônicas e sistemas de informação (OH, 1997). O estudo visou investigar que tipo de sistemas de informação em redes de alta velocidade permitiriam a acadêmicos usar recursos de informação de forma efetiva e eficiente. Segundo, questões relacionadas com a necessidade do tratamento confiável, eficiente e efetivo da informação no âmbito do Ministério das Relações Exteriores de Brunei (ABDUL-KARIM, 2001). E terceiro, questões relacionadas com a preservação de dados digitais, considerando o potencial do conceito de metadados como chave para a gestão universal de dados (CHILVERS, 2000).

\section{Estudos realizados no Brasil}

Buscas realizadas na literatura nacional apontam para um baixo índice de conhecimento da Metodologia no país, com a maior parte dos

\footnotetext{
${ }^{4}$ Espécie de check list que permite a definição de subsistemas relevantes de forma a incluir seus elementos fundamentais: Clientes, Atores, Transformação, W eltschauungen -visão de mundo, $\mathbf{O}$ wner - proprietário eEnvironmental constraints - restrições impostas pelo ambiente do sistema.

5 Espécie de cartoon, em que um exercício de estruturação do sistema (situação problema) em estudo é ilustrado. A ilustração resultante da análise da situação problema deve permitir, entre outros aspectos, a identificação de seus subsistemas relevantes.
} 
textos versando sobre suas características como exemplo de Metodologia ou de abordagem sistêmica. Exemplos de aplicação são encontrados nos trabalhos de Soares et al. (2001) e Ferrari et al. (2002), entre alguns outros poucos. O estudo coordenado por Ferrari, por exemplo, aplicado no ambiente de uma empresa distribuidora de produtos automotivos, visou a analisar o problema de como melhorar o desempenho da mesma. Há ainda, alguns ensaios na área agrícola, onde a mesma tem sido incluída em programas de treinamento e em dissertações (MARTINS 1996; PINHEIRO, 2002) e, ainda, um exemplo de discussão sobre o uso da Metodologia em ambiente acadêmico (SOARES, 1998), quando a autora analisa as relações entre a SSM e a Pesquisa-Ação, visando ressaltar as características da Metodologia como modalidade participativa.

Vale a pena ressaltar que, na área de Ciência da Informação no Brasil, a Metodologia parece ser totalmente desconhecida, a despeito dos exemplos de sua utilização em estudos de sistemas de informação e, como já mencionado anteriormente, em disciplinas de cursos e em projetos de tese, em outros países. Assim, apresentam-se, a seguir, algumas observações preliminares baseadas no seu ensino como disciplina específica em um programa de pós-graduação.

\section{O BSERV A ÇÕ ES PRELIM INARES SOBRE O ENSINO DESSM EM CIÊNCIA DA IN FOR M A ÇÃ O NOBRASIL}

Como disciplina específica, a Metodologia de Sistemas Flexíveis vem sendo oferecida pelo Programa de Pós-Graduação do CID desde 2001, dentro de uma série de disciplinas do programa denominada "Tópicos Especiais em Ciência da Informação", cujos conteúdos são variados. A experiência levou alguns alunos a decidirem por sua utilização em suas pesquisas, tanto de doutorado como de mestrado. Iniciou-se, igualmente, um processo de divulgação da mesma no âmbito do departamento, via apresentação de palestras como parte de outras disciplinas do programa e de módulos em disciplinas de cursos de especialização. Tem-se observado, até o momento, que a mesma vem despertando grande interesse por parte da maioria dos alunos, motivando-os para sua utilização tanto no ambiente de trabalho (suas próprias organizações) como em outras organizações e, ainda, em trabalhos acadêmicos.

Sabe-se que, o perfil dos estudantes do Programa de Pós-Graduação do CID é bastante diversificado. O curso recebe, portanto, alunos das mais variadas áreas do conhecimento, o que parece ser característico de programas de pós-graduação em Ciência da Informação em todo o mundo. O caso da disciplina em questão não é diferente: a mesma atrai alunos de várias áreas do conhecimento. Tal diversidade de backgrounds tem sido considerada como crucial para seu bom entendimento, discussão e aplicação em exercícios acadêmicos.

Lecionada por dois semestres verifica-se que, as áreas mais comuns dos alunos matriculados na disciplina foram até o momento: as Engenharias (de Comunicação, Civil, Elétrica, de Redes), Administração, Ciência da Computação, Comunicação, Arquitetura e Ciência da Informação. Vale ressaltar que a maior parte dos alunos, no entanto, tem sido das Engenharias e Ciência da Computação. Tal diversidade permitiu observar que é imprescindível a presença de profissionais e/ou pesquisadores dessas duas áreas (Engenharias e da Ciência da Computação) nas turmas, dado que a SSM, nestes casos, ao tempo em que guarda semelhança com as Metodologias típicas das mesmas, quebra alguns de seus paradigmas. Isto, por sua vez, provoca os mais diversos tipos de reação: de um questionamento crítico a uma aceitação natural das diferenças paradigmáticas introduzidas pela Metodologia no estudo sistêmico de problemas organizacionais. No entanto, ao 
interagir com a Metodologia, alunos de todas as áreas, especialmente dessas duas, têm-se mostrado altamente interessados no seu uso. Essas motivações levaram ao desenvolvimento, quando da primeira vez em que foi ensinada, de três projetos, desenvolvidos em grupo.

O primeiro envolveu uma situação problema da área de Engenharia Civil e visou identificar, descrever, estruturar e propor melhorias em questões preocupantes identificadas em uma empresa de engenharia. Tais preocupações compreendiam questões relacionadas com o departamento de marketing e vendas da empre$\mathrm{sa}$, as quais se mostravam como necessitando de uma intervenção tanto para serem melhor entendidas, quanto para serem melhoradas substancialmente. O trabalho apresentado tem norteado a atuação dos alunos envolvidos com o mesmo em suas atividades profissionais, usando a Metodologia.

O segundo, na área de Ciência da Informação, visava a discussão de questões relacionadas com o fluxo de atendimento ao cliente dos serviços de informação em uma empresa de pesquisa agrícola. Tal fluxo foi identificado como problemático em função da ocorrência de problemas como duplicações de tarefas, perda do conhecimento gerado pelo atendimento, falta de interação, trabalho dos setores envolvidos, entre outros. O trabalho continuou como pesquisa de mestrado e está em fase de conclusão. Alguns dos resultados preliminares estão sendo publicados na Revista de Biblioteconomia de Brasília (v.26, n.1, no prelo).

O terceiro abordou questões típicas da Administração e da Ciência da Informação, as quais se relacionam com a questão da gestão do conhecimento estratégico no âmbito de uma agência de regulação de serviços postais. Questões como a transformação de conhecimento tácito em explícito, o uso deste para geração de conhecimento estratégico e, consequentemente, para a proposição de ações estratégicas, nortearam o desenvolvimento do estudo. Parte deste trabalho está sendo usada como pesquisa de doutorado e os resultados do "exercício" estão sendo também publicados.

O ensino da Metodologia em uma segunda turma de alunos de pós-graduação tem-se concentrado na discussão de questões relacionadas com a comunicação científica e organizacional, além das questões de infra-estrutura tecnológica e adequação curricular, no âmbito do Departamento de Ciência da Informação da Universidade de Brasília. Tal estudo é, ainda, parte de um projeto de iniciação científica com alunos de graduação e deverá constituir estudo de caso da pesquisa de doutorado citada acima. Resultados bastante preliminares do estudo são apresentados a seguir.

\section{RESULTADOS PRIM IGÊ NIOS DE PESQUISA EM CIÊNCIA D A I NFORM A ÇÃ O NO BRASIL}

Os primeiros resultados do estudo acima citado são bastante preliminares e se baseiam em coleta de dados realizada via grupo focal em que professores do departamento que exercem função superior. Tais funções são: de chefe do departamento, de coordenação de graduação (Arquivologia e Biblioteconomia) e de coordenação de pós-graduação. Além desses, mais um professor sênior foi incluído na amostra, por dois motivos principais: primeiro, pela experiência tanto no âmbito do departamento quanto da faculdade em que o mesmo está inserido e em Conselhos da Universidade; segundo, na tentativa de minimizar a tendenciosidade da amostra por incluir somente professores detentores de função.

O grupo foi entrevistado pelos alunos da disciplina e pelos bolsistas do programa de iniciação científica sobre as quatro questões centrais, objeto de investigação. As respostas 
obtidas mostram que os tópicos estudados constituem, de fato, questões cruciais e preocupantes no departamento. $\mathrm{Na}$ interpretação daqueles "estudantes-pesquisadores", a situação pareceu, tal como discutida pelo grupo entrevistado, caótica. Isto, é bom lembrar, constitui situação ideal para aplicação da SSM.

Tal coleta de dados visou à aplicação do estágio 1 da Metodologia: situação problema mal estruturada. Isto é, as questões que preocupam os clientes, proprietários, solucionadores, etc., do problema em estudo, não estão suficientemente claras, o que, por sua vez, não permite a identificação daquilo que, de fato, constitui o problema ou o conjunto de problemas que compõem o que a Metodologia denomina "situação problema". Tais dados devem ser obtidos por meio do método de Pesquisa-Ação. Isto é, de Pesquisa Participante em que instrumentos de coleta como observação, questionários, entrevistas, grupo focal, análise documental, etc., são usados, dados são coletados e analisados, algumas intervenções na situação são realizadas, mais estudos são feitos a partir desses resultados, etc., formando um ciclo de aprendizagem sobre a mesma até que se chegue a um resultado que descreva suficientemente a situação (sistema). Até o momento, a questão que pareceu "pior estruturada" foi a comunicação organizacional. Mais e melhores detalhes dessa análise estarão disponíveis posteriormente. O desenvolvimento do estudo vai permitir, na verdade, confirmar ou não essa tendência.

Os dados obtidos nesse estágio da Metodologia estão sendo usados para a estruturação da situação problema investigada: estágio 2 da SSM: situação problema estruturada, na qual o sistema de atividade humana em estudo é definido, o que resulta na elaboração de uma "Ilustração Rica" (Rich picture). Nesta ilustração, os atores, processos, relacionamentos, hierarquias, áreas de conflito, observa- dores externos, questões que preocupam a todos os envolvidos interna ou externamente, entre outros aspectos, são representados. O trabalho está precisamente nesta fase, não sendo possível aqui incluir a llustração rica que vai resultar dessa etapa da análise.

\section{CO N S IDERAÇÕ ES FIN A IS}

As experiências descritas neste trabalho tornam oportuno observar como a utilização da SSM, em projetos de pesquisa na Ciência da Informação, pode contribuir para a discussão de questões típicas da área. Além disso, parece importante, também, avaliar sua contribuição para o desenvolvimento do corpo de conhecimentos da área, já que a mesma vem sendo objeto de pesquisa e estudos de pós-graduação. Mais que isso, parece relevante destacar como uma experiência com a Metodologia permitiu melhorar a interação da graduação com a pós-graduação em um departamento de uma universidade federal no Brasil. Destarte, a proposição de um programa de mudança organizacional, tal como descrito acima, deve contribuir para a discussão e o enriquecimento dessas questões.

É importante registrar que os resultados preliminares apresentados assim como os resultados da provável aplicação de todos os sete estágios da Metodologia ao longo do semestre em curso, deverão, ao final do mesmo, constituir o estudo-piloto da investigação realizada no âmbito do programa de iniciação científica. Mais que isso, conforme já dito anteriormente, parte desses resultados deverão constituir um estudo de caso na pesquisa de doutorado ora em andamento. O estudo principal, objeto do projeto de iniciação científica, deverá proporcionar, em última instância, o enriquecimento da interdisciplinaridade da Ciência da Informação, o desenvolvimento de seu corpo de conhecimentos e uma melhor interação entre a graduação e a pós-graduação no departamento, além de introduzir um conhecimento bastante novo neste ambiente. 


\section{REFERÊ N CIAS}

ABDUL-KARIM, H.S.H. Towards an integrated information system for a pubnlic sector organisation in Brunei Darussalam. Loughborough, 2001. Tese (Doutorado) - Loughborough University, Dep. of Information Science, 2001.

CHECKLAND, P. Systems thinking, systems practice. Chichester: John Wiley \& Sons,1999. p.166.

CHECKLAND, P.; SCHOLES, J. Soft systems methodology in action. Chichester: John Wiley \& Sons, 1999. p.29.

CHILVERS. H. Managing long-term access to digital data objects: a metadata approach 2000. Tese (Doutorado) - Loughborough University, Dep. of Information Science, 2000.

COUPRIE, D. et al. (CI) Soft systems methodology. Available from: <http://sern.ucalgary.ca/courses/ seng/613/F97/grp4/ssmfinal.html>. Acess: Jul. 2000.

FERRARI, F.M. et al. Uma aplicação da soft systems methodology à realidade de uma empresa brasileira. In: SEMEAD. Ensaio de administração geral. Disponível em:<http://www.ead.fea.usp.br/ Semead/AdmGeral.htm>. Acesso em: maio 2002.

HUSSERL, E. The idea of phenomenology. The Hague: M Nijhoff, 1970.
MARTINS, F.M. Aplicação da Metodologia multicritério de apoio à decisão na avaliação de políticas de gerenciamento em uma empresa agrícola. 1996. Dissertação (Mestrado) - Universidade Federal de Santa Catarina, Florianópolis, 1996.

$\mathrm{OH}, \mathrm{K} . \mathrm{M}$. Electronic networks and information services in South Korean universities. Loughborough, 1997. Tese (Doutorado).

PINHEIRO, S.L.G. O enfoque sistêmico e o desenvolvimento sustentável: uma oportunidade de mudança de abordagem hard system para experiências com soft systems. Disponível em: <http://www.emater.tche.br/docs/agroeco/revista/n2/ 08_artigo.htm>. Acesso em: abr. 2002.

SOARES, V.M.S. A soft systems methodology e a pesquisa-ação para projeto. In: SEMINÁRIO SOBRE METODOLOGIA DE PROJETOS DE EXTENSÃO. Extensão universitária e Metodologia participativa. Anais... Rio de Janeiro: COPPE/UFRJ, 1998.

SOARES, V.M.S. et al. Técnicas qualitativas e a soft system methodology aliadas ao enfoque sistêmico. Revista de Administração, v.36, n.3, p.95-99, 2001.

WILSON, B. Systems: concepts, metodologies, and applications. 2.ed. Chichester: John Wiley \& Sons, 2000. 
